\title{
Management of abnormal uterine bleeding by northern, rural and isolated primary care physicians: PART I - How are we doing? Simone N Vigod ${ }^{1}$ and Donna E Stewart*2
}

Address: ${ }^{1}$ University of Toronto, Faculty of Medicine, Canada and ${ }^{2}$ Women's Health, University Health Network, 657 University Ave., ML 2-004, Toronto, Ontario, M5G 2N2, Canada

E-mail: Simone N Vigod - simone.vigod@utoronto.ca; Donna E Stewart* - donna.stewart@uhn.on.ca

${ }^{*}$ Corresponding author

Published: 20 November 2002

BMC Women's Health 2002, 2:10

This article is available from: http://www.biomedcentral.com/1472-6874/2/10

(C) 2002 Vigod and Stewart; licensee BioMed Central Ltd. This is an Open Access article: verbatim copying and redistribution of this article are permitted in all media for any purpose, provided this notice is preserved along with the article's original URL.
Received: 14 June 2002

Accepted: 20 November 2002

\begin{abstract}
Background: Canadian hysterectomy rates have declined in recent years. However, hysterectomy rates for discretionary indications, principally abnormal uterine bleeding (AUB), remain high in some regions. In northern Ontario, hysterectomy rates for women aged 34-45 are almost triple the rates in southern, urban areas. Primary care physicians (family doctors) usually manage AUB initially in these northern areas where a severe shortage of gynecologists exists.

Methods: We surveyed 194 family physicians in northern Ontario with a case scenario of a premenopausal woman with heavy vaginal bleeding to characterize management and to gain physicians' perspectives on the factors that affect it.

Results: To investigate her heavy vaginal bleeding, only $17 \%$ of physicians recommended a pelvic examination for the woman in our case scenario. Most physicians advocated a course of medical therapy before referral to a gynecologist, for whom the average waiting time was seven weeks. However, most physicians recommended referral after only one failed trial of medical treatment. Physicians felt that major deterrents to medical treatments were patient desires for immediate relief and/or permanent solutions, poor patient compliance and the high cost of medication. Only $25 \%$ of respondents indicated that they would perform an endometrial biopsy prior to referral.

Conclusions: Family physicians would benefit from further education on appropriate investigations for AUB, primarily training in pelvic examination and endometrial biopsy techniques, as well as appropriate treatment algorithms. Further research into patient perspectives on treatment options is needed.
\end{abstract}

\section{Background}

Although hysterectomy rates have declined in developed countries over the past ten years, several countries including the United States, Canada and Australia have much higher rates than most European countries and report large variations in rates by region $[1,2]$. In Canada, for example, the age-adjusted hysterectomy rate for women aged 34-45 in some northern and rural areas of Ontario is nearly triple that in urban areas such as Toronto. The differences between regions are largely attributable to a higher rate of hysterectomies being performed for discretionary indications, primarily for abnormal uterine bleeding (AUB) that is unrelated to a systemic cause, anatomical lesion or malignancy [2]. Clinical practice 
guidelines conclude that hysterectomy is indicated primarily for women with AUB who have not had an adequate response to medical treatment or other surgical interventions [3-5]. The reasons for the increased regional hysterectomy rates are likely to involve physician, patient and economic factors. AUB is a common problem and accordingly primary care physicians (family doctors) are often responsible for its management, especially in northern and isolated areas where a severe shortage of gynecologists exists. It is essential that family doctors are confident in providing initial investigation and treatment for women who present with abnormal uterine bleeding. The present study characterizes the management patterns of primary care physicians in northern Ontario who responded to a survey and gains their perspectives on the factors that affect their management of AUB.

\section{Methods}

A five-page questionnaire entitled "Management of Heavy Vaginal Bleeding: Northern and Rural Primary Care Physicians" was developed in June, 2000 on the basis of the current accepted standards for the management of AUB and recommendations for hysterectomy (Keywords: Menorrhagia, Hysterectomy andAbnormal Uterine Bleeding) [6]. Clinical practice guidelines on AUB and hysterectomy were sought from the Society of Obstetricians and Gynecologists of Canada (SOGC) and the American College of Obstetricians and Gynecologists (ACOG). The guidelines available at the time of survey development had been published in 1996 (SOGC) and 1994 (ACOG) $[3,7,8]$. Input from family physicians, gynecologists, sociologists, psychologists and other women's health experts was incorporated during the survey development and piloting. The survey (see appendix - additional file 1) was composed of four parts, of which I. Demographic Information and II. Clinical Scenario, Investigations and Treatment are reported in this paper. Parts III. Clinical Practice Guideline Use and IV. Education and Resource Needs Assessment are described in the accompanying article ("Part II: What Do We Need?").

We collected information on physicians' sex, age, history of clinical practice, Internet access (for availability of information), previous obstetrical experience (possible indication of comfort level with managing gynecological problems) and Canadian College of Family Physicians (CCFP) membership status (requirements for continuing medical education (CME) and access to CME resources).

In the clinical scenario, a 39 year old woman presents with heavy cyclic vaginal bleeding of 3 months duration which impacts her daily function. Family physicians were asked what investigations they would perform and assuming that the results of all investigations were normal (i.e. that the cause of the bleeding was not identifiable), to outline their first, second and third lines of treatment.

The questionnaire was mailed to 686 physicians in the northern Ontario regions in the three district health councils that have the highest rates of hysterectomy[2]. Physicians were identified with the help of the Northern Shores District Health Council, the College of Physicians and Surgeons of Ontario (CPSO) web page and the local Yellow Pages. Each package included a cover letter explaining the objectives of the study, a questionnaire and a stamped return envelope. A modification of the Dillman (TDM) approach was used with two reminders, sent 3 and 8 weeks respectively after the original, each including a replacement questionnaire and a reminder letter [9]. Fiftyfour surveys were returned due to incorrect address or specialty information (i.e. respondents were not family physicians). There were 194 completed responses from the remaining 622 potential participants (Response Rate $=$ $31 \%)$. Response rates were evenly distributed among the three health regions and all respondents were family physicians currently practicing in northern, rural and/or isolated areas.

The Statistical Package for the Social Sciences (SPSS, version 10) was used for statistical analysis. Investigation and treatment recommendations generated by physicians were coded into individual variables. Descriptive analyses of all continuous and categorical variables were conducted. Chi square tests of independence investigated whether responses were dependent on sex, Internet use, previous obstetrical experience or Canadian College of Family Physicians (CCFP) membership status. Independent sample t-tests and analyses of variance (ANOVA) were used to determine any differences in responses by age or the population of the physician's practice location.

\section{Results}

\section{Physician information}

Responding physicians, mean age $43.4+/-10.5$ years, had been in family practice an average of $14.9+/-10.8$ years. Corresponding to the gender distribution of physicians in Ontario, $37.5 \%$ of the respondents were female, $62.5 \%$ were male. Males were significantly older with more years in family practice than females $(\mathrm{t}=-4.612, \mathrm{p}=0.00 ; \mathrm{t}=$ $4.324, \mathrm{p}=0.00$ ). All physicians practiced in northern Ontario, with $45.1 \%$ also designating their practice populations as "rural" and $17.5 \%$ as "isolated". These categories were not mutually exclusive (i.e. a physician could practice in an area that is rural and isolated). Fifty percent of physicians were practicing in areas with populations of less than 15000 people, with the remainder in larger towns $(25 \%)$ or small cities $(25 \%)$. Three-quarters of the respondents were members of the Canadian College of Family Physicians (CCFP) indicating completion of a 
family practice residency, successful passing of an examination in family practice and participation in continuing medical education (CME). A higher proportion of women were members of the CCFP than men in keeping with their younger age $\left(\chi^{2}=13.8, p=0.00\right)$. With respect to obstetric experience, $41.4 \%$ had previously delivered babies in their practices, $32.5 \%$ were currently doing deliveries and $26.2 \%$ had never delivered babies in their practices. Almost all (92.7\%) respondents had Internet access either in their homes or offices.

\section{Investigations and treatment}

Investigations suggested by physicians are reported in Table 1 . The tests recommended most frequently were pelvic ultrasounds $(87.1 \%)$, complete blood counts $(75.3 \%)$ and thyroid function tests $(41.2 \%)$. Endometrial biopsy (EB) was recommended by $25.3 \%$, and dilatation and curretage (D\&C) by $24.2 \%$, of physicians either during the initial investigation or after treatment failure. Older physicians were more likely to recommend $D \& C$ than younger physicians $(\mathrm{t}=2.28, \mathrm{p}=0.02)$ and women physicians were more likely to recommend EB than men $\left(\chi^{2}\right.$ $=5.13, p=0.02)$. Seventeen percent of physicians indicated that they would perform a pelvic exam on the patient in the clinical scenario, with a greater proportion of nonCCFP members recommending pelvic exams than CCFP members $\left(\chi^{2}=6.83, \mathrm{p}\right.$ 0.009). Some respondents $(15.5 \%)$ recommended coagulation testing. These physicians were significantly younger than physicians who did not order the aforementioned tests $(\mathrm{t}=-2.51, \mathrm{p}=0.015)$.

Table 2 presents physician treatment patterns for first, second and third lines of treatment. Medical options were the most highly recommended first and second lines of treatment. Physicians who chose medical options as a first line of treatment were significantly younger than those who did not (mean age 42 versus $50, t=-4.1, p=0.00$ ) and had been in family practice for less time (13.6 versus 22.5 years, $\mathrm{t}=-4.1, \mathrm{p}=0.00)$. Oral contraceptives $(54 \%)$ and non-steroidal anti-inflammatory drugs $(26 \%)$ were the most popular recommendations. A second trial of medical therapy was recommended most frequently by female physicians and CCFP members. Family doctors who practice obstetrics were more likely than those who did not, to recommend a third trial of medical therapy $\left(\chi^{2}=6.47, \mathrm{p}\right.$ $=0.01)$.

Physicians who recommended immediate referral to a gynecologist $(9.3 \%)$ were significantly older, and had been in practice longer, than those who did not. Male physicians and non-CCFP members were more likely to refer after the failure of only one trial of medical treatment. Upon failure of a second medical treatment, $84 \%$ of physicians who had not yet referred the patient to a gynecologist did so. Of these referring physicians, $20 \%$ specif-
Table I: Investigations: Physician Recommendations ( $=194)$

\begin{tabular}{lc}
\hline Investigation & N (\%) \\
\hline & \\
Pelvic ultrasound & $169(87.1)$ \\
Complete blood count (cbc) & $146(75.3)$ \\
Thyroid function tests & $80(41.2)$ \\
Endometrial biopsy* & $49(25.3)$ \\
Iron and/or serum ferritin & $48(24.7)$ \\
Dilatation and curettage* & $47(24.2)$ \\
International normalized ratio & $46(23.7)$ \\
(INR)/ prothrombin time (PT) & \\
Platelets & $46(23.7)$ \\
Pap smear & $45(23.2)$ \\
Pelvic exam & $33(17.0)$ \\
Partial thromboplastin time & $30(15.5)$ \\
Follicle stimulating hormone (FSH) & $22(11.3)$ \\
Luteinizing hormone (LH) & $18(9.3)$ \\
Glucose & $10(5.2)$ \\
Abdominal exam & $8(4.1)$ \\
Estrogen & $8(4.1)$ \\
Pregnancy test (beta HCG) & $8(4.1)$ \\
Prolactin & $7(3.6)$ \\
Progesterone & $4(2.1)$ \\
Referral (no investigations) & $4(2.1)$ \\
&
\end{tabular}

* requested either during initial investigation or during course of treatment

ically recommended hysterectomy (note that physicians were not asked to speculate on the outcome of a referral). Average waiting time for a gynecological referral for a woman with AUB was approximately 7 weeks (range $=28$ weeks) and more than $50 \%$ of patients would have to commute for at least one hour to attend a gynecologist.

Physicians identified several patient factors as deterrents to medical and/or hormonal management of AUB (Table 3). Major deterrents to medical management were a desire for immediate relief $(70.8 \%)$, poor compliance $(51.9 \%)$ and that it might not be a permanent solution (49.7\%). Another deterrent was financial impact on patients $(31.9 \%)$ and this was cited more often by physicians who practiced in areas with smaller populations $(\mathrm{t}=-3.30, \mathrm{p}=$ $0.001)$.

\section{Discussion}

The study's response rate (31\%) illustrates one of the greatest challenges in improving the management of AUB, that is, to communicate with all family physicians who manage this problem, both to determine their needs and to disseminate information. Current lists of active family doctors practicing in the North are difficult to obtain as there is a rapid turnover of physicians and this presents a challenge for surveys and education. The physicians who 
Table 2: Treatment Pattern for AUB.

\begin{tabular}{llll}
\hline Treatment & $\begin{array}{l}\text { First Line } \\
\text { Frequency (\%) }\end{array}$ & $\begin{array}{l}\text { Second Line } \\
\text { Frequency (\%) }\end{array}$ & $\begin{array}{l}\text { Third Line } \\
\text { Frequency (\%) }\end{array}$ \\
\hline Watchful Waiting & $7(3.6)$ & $1(0.5)$ & $0(0)$ \\
Medical & $105(54)$ & $51(28)$ & $3(2.4)$ \\
Oral Contraceptive & $50(26)$ & $8(4.4)$ & $1(0.8)$ \\
NSAID & $22(11)$ & $33(18)$ & $6(4.8)$ \\
Progesterone alone & $2(1.0)$ & $0(0)$ & $0(0)$ \\
Estrogen alone & $2(1.0)$ & $2(1.1)$ & $4(3.2)$ \\
Anti-fibrinolytic & $3(1.5)$ & $6(3.3)$ & $6(4.8)$ \\
Gonadotropin Agonist & $18(9.3)$ & $88(48)$ & $106(84)$ \\
Referral & & $31(17)$ & $10(7.9)$ \\
Surgical & $6(3.1)$ & $10(5.5)$ & $13(10)$ \\
Dilatation and Curettage & $1(0.5)$ & $10(5.5)$ & $25(20)$ \\
Endometrial Ablation & $2(1.0)$ & 183 & 126 \\
Hysterectomy & 194 & &
\end{tabular}

Table 3: Deterrents to Medical or Hormonal Management of AUB: Patient Factors $(\mathbf{N}=185)$

\begin{tabular}{lc}
\hline Deterrent & N (\%) \\
\hline & \\
Desire for immediate relief & $131(70.8)$ \\
Poor compliance & $96(5 \mathrm{I} .9)$ \\
May not be a permanent solution & $92(49.7)$ \\
Financial impact & $59(3 \mathrm{I} .9)$ \\
Limited patient comprehension & $52(28.1)$ \\
Patient's partner's preferences & $23(12.4)$ \\
Distance patient lives from medical care & $15(8.1)$ \\
Transportation problems & $13(7.0)$ \\
Embarrassment or offence of patient & I2 (6.5) \\
Other: contraindications, side effects, cultural/ & $\mathrm{N} / \mathrm{A}$ \\
religious beliefs, family planning, health beliefs (i.e. & \\
that medication won't work) & \\
\end{tabular}

responded to this questionnaire may be more highly motivated and a more geographically stable group than the average. As three-quarters of them were members of the College of Family Physicians they accordingly may represent a more highly trained, younger and possibly more knowledgeable subset of the practicing regional general practitioners. With this in mind, the authors take caution in interpreting the results of this questionnaire. However, as physicians who are practicing medicine in the northern, rural and isolated regions are all affected by problems such as time pressure and lack of resources, the issues identified by our respondents may be the tip of the iceberg.
To identify areas where responding physicians can improve their management of AUB, we compared their recommended investigation and treatment responses to best evidence guidelines or algorithms available at the time that the survey was conducted. Since this time, clinical practice guidelines on the investigation and management of AUB have been updated [5,6]. However, as the majority of respondents were unaware of any existing guidelines at the time of our survey, it is unlikely that they have significantly changed their practices in light of the new ones (see accompanying article).

Current clinical practice guidelines (CPGs) recommend a pelvic exam to visualize the cervix and palpate pelvic masses, however, only $17 \%$ of respondents indicated that they would do this. These physicians may not be confident in their pelvic exam skills or perhaps believe that a pelvic exam can be replaced by pelvic ultrasound (suggested by $87 \%$ of physicians). Pelvic exam training and identification of cervical lesions and uterine fibroids would allow further specific investigation and treatment to be instituted earlier for affected women thereby decreasing the need for unnecessary investigations and costs to the patients and the health care system.

Appropriately, the majority of family physicians suggested a complete blood count to look for an anemia. Thyroid function tests, recommended by almost half of the responding physicians, and coagulation tests (recommended by $15.5 \%$ ) should be considered when there is clinical suspicion of thyroid dysfunction or bleeding disorders respectively. Identification and appropriate treatment of thyroid dysfunction and bleeding disorders may reduce the necessity for surgical intervention in AUB. Tests of fol- 
licle stimulating hormone (FSH) and luteinizing hormone (LH), recommended by $11.3 \%$ and $9.3 \%$ of respondents respectively, are more useful in the clinical context of examining ovarian function.

Whether endometrial sampling to look for endometrial hyperplasia or neoplasia was necessary in this scenario is a controversial point. Guidelines in existence at the time of the study stated that endometrial sampling should be done in any pre-menopausal woman with heavy vaginal bleeding who is refractory to treatment [3]. More specifically, recent guidelines (2002) support endometrial sampling in premenopausal women with AUB who are over forty years of age, who have risk factors for endometrial cancer including new onset of heavy, irregular bleeding or whose bleeding is refractory to three months of medical therapy [4]. As the woman in our case scenario had cyclic bleeding, endometrial sampling may not have been necessary in the initial management plan, yet less than half the respondents recommended endometrial sampling at any time during her management, even after she did not improve with treatment.

Endometrial biopsy (EB) is the initial diagnostic endometrial sampling procedure of choice for detecting endometrial hyperplasia or neoplasia (sensitivity 67-95\%). Diagnostic D\&C has a lower sensitivity and higher procedural risk (e.g. uterine perforation, hemorrhage, anesthetic complications) and it is only recommended when no other sampling methods are feasible. Pelvic ultrasound is neither sensitive nor specific for endometrial carcinoma in premenopausal women and can therefore not be used as a form of endometrial sampling [10]. EB can be performed in-office by family physicians and having the ability to rule out endometrial hyperlasia and neoplasia will allow medical treatments to be attempted, possibly reducing gynecological referral rates in the long-term.

Most respondent physicians advocated for at least one course of medical therapy before referring the patient to a gynecologist and/or recommending surgical intervention. It is not surprising that younger family doctors were most likely to recommend initial medical treatment as they have the greatest proximity to their medical training and AUB management has changed in recent years. Further, CCFP members (who have continuing medical education requirements) and those practicing obstetrics (who likely have a high proportion of women in their practices and may be more confident and up-to-date on obstetrical and gynecological issues) were most likely to recommend subsequent medical treatments. This highlights the impact of knowledge and confidence on physician management patterns.
Many physicians reported that patient desire for immediate relief and permanent solutions were deterrents to medical or hormonal treatment. However, the waiting time for a gynecological referral could encompass several cycles of heavy bleeding and missed work. By this point, an untreated woman experiencing significant frustration and distress may have an increased wish for a definitive treatment, such as hysterectomy [11]. Poor compliance was anticipated as a deterrent to medical management by over half of responding physicians. Therefore, it is important to educate women about the medications and their effects. Physician perceptions of patient deterrents to medical management must be further confirmed with patients in order to design appropriate interventions.

\section{Conclusions}

Evidence and opinion on the management of AUB changes rapidly and the efforts in the management of AUB by already overburdened primary care physicians in northern, rural and isolated areas should be commended. However, family physicians would benefit from further education on appropriate investigations and treatment for AUB, training and practice in pelvic exam and endometrial biopsy techniques. As well, women may not have all of the facts about medical and surgical treatment options and this is an opportunity for primary care physicians to help women obtain the best information to make effective treatment decisions. Specific suggestions for the form and dissemination of these recommendations are found in the accompanying article.

\section{Competing interests}

None declared.

\section{Authors' contributions}

Dr. Stewart conceived of the project, supervised the work, and fine-tuned the write-up. Ms. Vigod conducted the literature review, developed the questionnaire, conducted the survey, analyzed the data and drafted the report.

\section{Appendix Clinical scenario}

A 39-year old woman presents with a three-month history of cyclic, heavy vaginal bleeding every 24 days. She is without pain, bowel or bladder complaints. However, her heavy bleeding prevents her from going to work 4-6 days per cycle. She has no history of medical problems and is not taking any medications. She has only been hospitalized once, for the uncomplicated birth of her only child, 3 years ago. For the past 5 years, on pelvic examination, she has had a fibroid the size of an 8-week pregnancy.

\section{Acknowledgements}

We are grateful to Dr. Leyland for his suggestions on this project and manuscript. 
Nicholas A. Leyland, MD, FRCSC

Head of Gynecology

Mount Sinai Hospital

Department of Obstetrics and Gynecology

University of Toronto

\section{References}

I. Reid BA, Aisbett CW, Jones LM: Relative utilization rates of hysterectomy and links to diagnoses. Canberra: Publications Production Unit (Public Affairs, Parliamentary and Access Branch), Commonwealth Dept of Health and Aged Care 1999

2. Cohen $M$, Hall R: Variations in selected surgical procedures and medical diagnoses by year and region - hysterectomy. In: Patterns of Health Care in Ontario - The ICES Practice Atlas Ottawa: Canadian Medical Association 1996

3. Society of Obstetricians and Gynecologists of Canada: Clinical practice guidelines for hysterectomy (policy statement). J Soc Obstet Gynecol Can 1996, 47:

4. Vilos GA, Lefebvre G, Graves GR: Guidelines for the management of abnormal uterine bleeding. SOGC Clinical Practice Guidelines 2001, 106:

5. Lefebvre G, Allaire C, Jeffrey J, Vilos G: Hysterectomy. SOGC Clinical Practice Guidelines 2002, I 09:

6. Broder MS, Kanouse DE, Mittman BS, Bernstein SJ: The appropriateness of recommendations for hysterectomy. Obstet Gynecol 2000, 95:199-205

7. ACOG: Technical Bulletin: Dysfunction uterine bleeding. Washington: American College of Obstetricians and Gynecologists 1989

8. ACOG: Quality assessment and improvement in obstetrics and gynecology: ACOG criteria sets. Washington: American College of Obstetricians and Gynecologists 1994

9. Dillman DA: Mail and Internet surveys: The tailored method. New York, John Wiley and Sons 1999

10. Brand A: Diagnosis of endometrial cancer in women with abnormal vaginal bleeding. J Soc Obstet Gynecol Can 2000, 22:102-4

II. Geller SE, Bernstein S], Harlow SD: The decision-making process for the treatment of abnormal uterine bleeding. $J$ Womens Health 1997, 6:559-67

\section{Pre-publication history}

The pre-publication history for this paper can be accessed here:

http://www.biomedcentral.com/1472-6874/2/10/prepub

Publish with BioMed Central and every scientist can read your work free of charge

"BioMed Central will be the most significant development for disseminating the results of biomedical research in our lifetime. "

Sir Paul Nurse, Cancer Research UK

Your research papers will be:

- available free of charge to the entire biomedical community

- peer reviewed and published immediately upon acceptance

- cited in PubMed and archived on PubMed Central

- yours - you keep the copyright

Submit your manuscript here:

http://www.biomedcentral.com/info/publishing_adv.asp
BiolMedcentral 